\title{
Prevención de infecciones respiratorias. Situación en 12 jardines maternales privados de la Ciudad Autónoma de Buenos Aires
}

\author{
Prevention of respiratory tract infections. Situation in \\ 12 private day care centers in the city of Buenos Aires
}

\author{
Dra. María Elina Serra ${ }^{a}$ y Prof. Lic. Rose Mari Soria
} RESUMEN
Introducción. La asistencia al jardín maternal
es un factor de riesgo para contraer infecciones
respiratorias. Existen medidas de prevención
que deberían aplicarse.
Objetivos. Describir la disponibilidad de
insumos o infraestructura edilicia apropiados;
hábitos, conductas y capacitación del personal;
y la existencia y aplicación de recomendaciones
oficiales o normas institucionales para la
prevención de infecciones respiratorias en
jardines maternales de la Ciudad de Buenos
Aires.
Métodos. Estudio exploratorio observacional
transversal realizado en jardines maternales
privados seleccionados en forma sistemática. Se
realizóuna entrevistaalresponsableinstitucional
y una sesión de observación de tres horas de
duración en una sala de cada jardín. Se recabó
información sobre lavado de manos, higiene de
secreciones nasales, pautas de exclusión escolar,
control de inmunizaciones, promoción de
lactancia materna y existencia de guías, normas
y capacitación sobre prevención de infecciones
respiratorias.
Resultados. Se incluyeron 12 jardines. Si bien en $8 / 12$ salas se contaba con infraestructura y elementos necesarios, el lavado de manos fue infrecuente y con técnica inadecuada. La higiene de secreciones nasales se realizó sin higiene de manos posterior. En 11/12 jardines, se realiza control de las inmunizaciones de los niños; en 9/12, no se cumplen las pautas de exclusión escolar; en ninguno hay material de promoción de la lactancia materna. En 1/12, los docentes habían recibido capacitación sobre el tema en el último año y, en 1/12, había una guía propia para prevención de infecciones.

Conclusión. Pese a la disponibilidad de recursos, la implementación de medidas de prevención no se realizó en forma regular y sistemática en los jardines visitados.

Palabras clave: jardines infantiles, infecciones respiratorias, prevención de infecciones.

Financiamiento:

Este trabajo ha sido financiado por FUNDASAMIN.

Conflicto de intereses: Ninguno que declarar.

Recibido: 20-11-2014 Aceptado: 30-1-2015 importante de internación, luego de las malformaciones y los problemas perinatales y la principal causa de mortalidad en los menores de un año. ${ }^{1-4}$

La asistencia al jardín maternal es un factor de riesgo conocido para contraer infecciones. Su relevancia reside en las implicancias para el niño y en la diseminación de estas enfermedades dentro de la comunidad. 5,6

Los cambios socioculturales que derivan de la inclusión de las madres en el mercado laboral han generado la necesidad de cuidado de los niños fuera de su hogar. ${ }^{7-9}$

Si bien existen medidas de prevención, tales como el lavado de manos, la adecuada higiene de secreciones, la exclusión de niños y adultos sintomáticos o la promoción de la lactancia, en nuestro país, como en muchos otros, no se cuenta con guías sobre el tema dirigidas al personal de centros de cuidado infantil. ${ }^{10}$ Los jardines privados de la Ciudad Autónoma de Buenos Aires (CABA) se rigen por la Ley 621, que contiene disposiciones generales sobre aspectos vinculados a la salud, pero, excepto la exigencia de pileta exclusiva en sectores separados para alimentación y cambio de pañales, no menciona otros procedimientos o medidas de prevención. ${ }^{11}$ De este modo, quienes pueden implementar medidas podrían no conocerlas, no saber cómo hacerlo o no poder ponerlas en práctica.

Se desarrolló un estudio exploratorio de la situación en jardines maternales privados de la CABA. 


\section{OBJETIVOS}

1. Describir la disponibilidad de elementos, insumos o infraestructura edilicia apropiados para evitar la diseminación de las infecciones respiratorias.

2. Describir los hábitos/conductas y capacitación del personal relacionados con la prevención de infecciones respiratorias en los jardines maternales.

3. Determinar la existencia y aplicación de directivas de entidades oficiales o normas institucionales para la prevención de infecciones respiratorias.

\section{METODOLOGÍA}

Se realizó un estudio descriptivo de diseño observacional transversal en 12 jardines maternales de gestión privada de la CABA.

\section{Selección de los jardines participantes}

Se dividió a la ciudad en 12 áreas geográficas. Se obtuvo el listado de jardines con registro definitivo de la Dirección de Educación de Gestión Privada. ${ }^{12}$ Se clasificaron los jardines por zona y se ordenaron alfabéticamente. Se contactó por teléfono de acuerdo con el orden de cada zona. Se realizaron hasta 3 llamados, con frecuencia semanal a cada jardín, antes de pasar al siguiente del listado. Se visitó el primero que accedió a participar de cada zona.

Los criterios de inclusión fueron los siguientes:

- El jardín debía incluir en su población a niños de entre 45 días y 2 años de edad.

- El jardín debía estar inscripto en el Registro de Instituciones Educativas Asistenciales de la Dirección General de Escuelas de Gestión Privada y/o Dirección de Educación Inicial del Gobierno de la Ciudad de Buenos Aires.

- La autoridad competente a cargo del jardín maternal debía consentir la realización del estudio por escrito.

Se consideró como criterio de exclusión que el jardín se encontrase emplazado en una institución dedicada a la atención de la salud o dependiese de ella por tratarse de un posible sesgo.

Durante la primera visita, se realizó el proceso de consentimiento informado a los directivos del jardín. Se explicó en detalle el estudio, se contestaron las dudas y se procedió a la firma del formulario de consentimiento informado.

Posteriormente, en cada jardín, se realizaron una serie de procedimientos:

a) Entrevista semiestructurada al responsable institucional (o a la persona delegada por el responsable).
El formulario de recolección de información para las entrevistas fue elaborado por las autoras, revisado por un profesional especialista en investigación cualitativa y ajustado, mediante entrevistas piloto con coordinadoras y directoras de jardines.

b) Sesión de observación de 3 horas de duración en una sala de menores de dos años (a elección del responsable institucional).

Las acciones sobre las que se obtuvo información a través de entrevista y observación fueron las siguientes (Anexos 1 y 2, en formato electrónico):

- Lavado de manos (en el personal y los niños; pautas establecidas para realizarlo, disponibilidad de elementos y realización correcta del procedimiento).

- Higiene de secreciones nasales.

- Higiene de elementos (modo y frecuencia).

- Cambio de pañal (por constituir un marcador relevante de pautas de higiene).

- Exclusión escolar (pautas establecidas).

- Disponibilidad de recursos (condiciones físicas, ambientales, etc.).

- Control de inmunizaciones de niños y del personal.

- Condiciones de la institución que promuevan la lactancia materna.

- Existencia de guías o normas sobre prevención.

Todas las visitas fueron realizadas por las autoras. Luego de cada una de ellas, se elaboró un informe escrito confidencial dirigido a los responsables de cada institución con el diagnóstico de situación y sugerencias para implementar mejoras. Se desarrolló un taller sobre prevención de infecciones respiratorias con todo el personal de cada jardín luego de la entrevista y la observación.

La información se ingresó a una base de datos creada para tal fin.

\section{Análisis estadístico}

Se realizó un análisis descriptivo de las variables registradas. Las variables cualitativas se analizaron a través de frecuencias y porcentajes, y las variables cuantitativas, a través de medidas de resumen de tendencia central y dispersión. Se utilizó el programa Excel 2007.

\section{Aspecto regulatorio y ético}

Este estudio fue evaluado y aprobado por el Comité de Ética de CEMIC.

En todas las instituciones participantes, se realizó el proceso de consentimiento informado 
con el responsable institucional y se pusieron a su disposición todos los documentos referentes al estudio para presentar ante las autoridades pertinentes. Se facilitó a las instituciones participantes un modelo de comunicación dirigido a los padres para informarles sobre la realización del estudio. Se mantuvo la confidencialidad de los datos y el anonimato de las instituciones participantes. La base de datos fue registrada en la Dirección Nacional de Protección de Datos Personales.

\section{RESULTADOS}

Se incluyeron 12 jardines (Figura 1) entre mayo y agosto de 2013. Se entrevistaron 7 directoras y 5 coordinadoras. La mediana de tiempo de funcionamiento de los jardines participantes fue 12,5 (2-48) años. La mediana de niños menores de dos años que concurrían fue 26 (16-85). En 4 jardines, concurrían niños con condiciones de salud que predisponían a infecciones respiratorias, tales como prematurez, antecedentes de patología pulmonar grave al nacer o cardiopatías. En 8 jardines, hubo 23 niños internados por infección respiratoria (mediana de 2; rango de 1-6) durante el último año.

Las observaciones se realizaron en 1 sala de lactantes, en 3 de lactantes y deambuladores y en 8 exclusivas para deambuladores. La mediana de niños por sala fue de 9 (4-12), la mediana de maestros fue de 2 (1-2) y la mediana de la relación niños/maestro fue de 5 (3-9).

En todos los jardines, los directivos consideraron que las infecciones respiratorias eran frecuentes entre los niños. En 7/12, refirieron que estas infecciones solamente eran peligrosas para niños con factores de riesgo. Cuando se interrogó acerca de la epidemia de gripe A (2009), en 7 jardines, se refirió haber implementado o enfatizado el uso de alcohol en gel como medida de prevención. En los restantes 5 jardines, no hubo cambios respecto a pautas de prevención.

En la Tabla 1, se presentan los datos sobre infraestructura y equipamiento de las 12 salas observadas.

En la Tabla 2, se expone lo observado sobre hábitos de higiene. La frecuencia de higiene de manos luego de la limpieza de secreciones nasales fue prácticamente nula. Además, se observaron 10 situaciones en que los niños presentaron secreciones nasales visibles y no se realizó higiene de ellas. En ninguno de los jardines, se observó material gráfico de promoción sobre higiene de manos. Solamente en dos de las salas observadas, los adultos tenían ropa de uso exclusivo para la sala y, en otras dos, calzado de uso exclusivo. En 10/12 salas observadas, al menos una de las maestras lucía accesorios en las manos y/o antebrazos. La capacitación del personal y la existencia de guías fue infrecuente (Tabla 3).

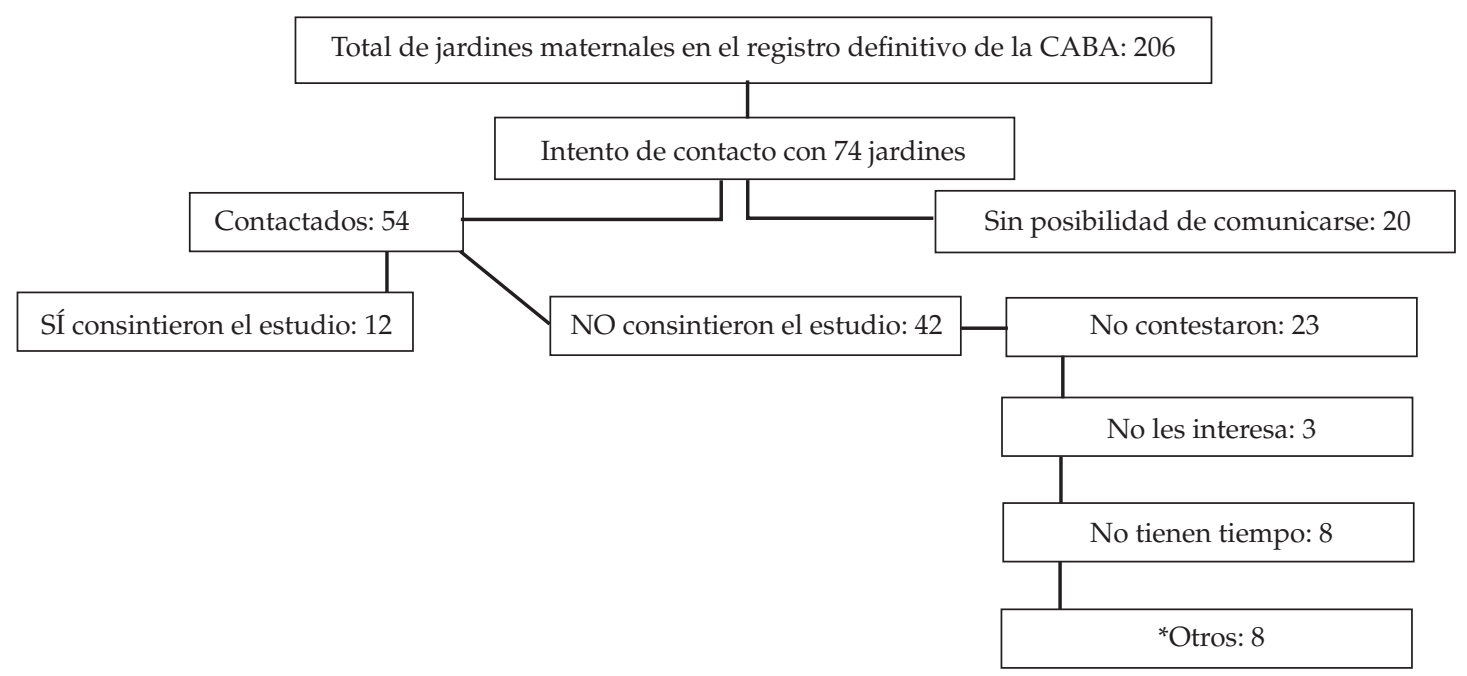

* Se niegan a consentir la realización del estudio luego de haber aceptado la visita por miedo a ser denunciados y/o tener otro concepto de salud-enfermedad. 
En 4 jardines, se refirió u observó el uso de aerosoles desinfectantes o aromatizantes.

En todos los jardines visitados, se llevaba un registro de la vacunación de los niños. En 11/12, se controlaba la vacunación del personal, pero tanto las inmunizaciones requeridas como los criterios de indicación fueron heterogéneos.

En 8/12 jardines, no existía indicación formal para que un maestro se retirara de una sala por problemas de salud. En 9 instituciones, se refirió que la mayor parte de los padres llevaban a sus hijos al jardín aunque estuvieran enfermos y argumentaban que preferían que los llamaran a sus trabajos si la situación empeoraba en lugar de no concurrir a trabajar. Al preguntar los motivos de salud frente a los cuales se llamaba a los padres para que retiraran a sus hijos, solo en un jardín se mencionó la dificultad respiratoria. En 7/12 jardines, se llevaba un registro de ausencias por motivos de salud; en 8 , se pedía certificado médico para reintegrarse luego de una enfermedad.

En cuanto a la lactancia, en 5/12 jardines, las madres dejaban leche materna para sus hijos; en 3, refirieron que concurrían a amamantar $y$, solamente en uno de los jardines, existía un área específica destinada a ello. En ninguno de los jardines, se observó material gráfico de promoción de la lactancia materna.

Tres de las instituciones contaban con un profesional de la salud que desarrollaba tareas de asesor.

\section{DISCUSIÓN}

En la mayoría de las instituciones visitadas durante este estudio, hubo una implementación deficiente de medidas de prevención de infecciones respiratorias, pese a que se disponía de los recursos adecuados en gran parte de ellas.

El lavado de manos, la medida de prevención inespecífica con mayor evidencia, ${ }^{13-15}$ un procedimiento sencillo y factible de implementación, no se puso en práctica en la mayoría de las situaciones que lo hubiesen requerido. Más aún, en los casos en los que se realizó, muchas veces, no se aplicó el procedimiento en forma correcta.

Estos resultados coinciden con los hallados por un estudio holandés que incluyó 122 centros de cuidado infantil, ${ }^{16}$ en los que el cumplimiento general de lavado de manos fue del $42 \%$, y con el 16\% de lavado de manos luego del cambio de pañales informado por Barros y col. en Brasil. ${ }^{17}$

TABla 1. Aspectos edilicios y equipamiento de las salas observadas

\begin{tabular}{|c|c|c|}
\hline Planta física & SÍ & Comentarios \\
\hline Ventanas al exterior & 10 & * La terraza es un sitio usado por los fumadores \\
\hline Aire acondicionado & 4 & a pesar de que es parte de las instalaciones del jardín \\
\hline Calefacción por combustión & 0 & donde rige la prohibición de fumar. \\
\hline Piso lavable & 9 & \\
\hline Alfombras removibles & 5 & \\
\hline Área para fumadores* & 0 & \\
\hline Recursos de higiene & SÍ & Comentarios \\
\hline Pileta en sala & 9 & * En dos casos, hay crema de manos \\
\hline Canilla monocomando & 4 & de uso común sin dosificador. \\
\hline Jabón líquido & 8 & ** Se observó recipiente para residuos \\
\hline Jabón en barra & 2 & con tapa pero sin pedal en 7 salas. \\
\hline Papel para secado & 8 & \\
\hline Alcohol en gel accesible en dispositivo expendedor & 11 & \\
\hline Crema de manos en dispositivo expendedor* & 0 & \\
\hline Elementos individuales para higiene (ej.: óleo) & 2 & \\
\hline Pañuelos descartables accesibles & 9 & \\
\hline Guantes & 10 & \\
\hline Bolsitas individuales para descarte & 4 & \\
\hline Recipiente para residuos con tapa y pedal** & 0 & \\
\hline Otros recursos & SÍ & Comentarios \\
\hline Cunas individuales y lavables & 2 & * En 3 salas, se observó el uso de colchonetas no individuales. \\
\hline Almohadones y/o almohadas & 5 & ** En la práctica, no se usan en forma exclusiva. \\
\hline Colchonetas individuales y lavables* & 4 & \\
\hline Sector pañales y alimentación exclusivos ${ }^{* *}$ & 10 & \\
\hline Materiales gráficos de prevención & 0 & \\
\hline
\end{tabular}


Las situaciones de contacto con secreciones respiratorias no fueron manejadas como potencialmente infecciosas. Pese a que, la mayoría de las veces, se utilizaron pañuelos de papel descartables, lo que ha sido asociado con un riesgo menor de infección por gérmenes respiratorios, como Haemophilus influenza, ${ }^{18}$ la higiene posterior de manos ha sido inconstante entre el personal y nula para los niños, lo que coincide también con el hallazgo de Zomer, ${ }^{16}$ quien constató que solo en un $25 \%$ de estas situaciones se realizaba algún tipo de higiene posterior.

La relación entre cantidad de niños por maestro, un factor que se ha relacionado con una menor tasa de higiene de manos, ${ }_{1}^{16}$ fue adecuada y acorde a lo establecido en la Ley 621 en la mayoría de las salas visitadas.

La limpieza y desinfección del ambiente, una medida efectiva para disminuir la propagación de gérmenes, ${ }^{19,20}$ no sigue una sistemática. El desconocimiento y la falta de normativa podrían dar lugar a la toma de medidas que, además de no tener fundamento desde el punto de vista científico, resultaran contraproducentes, tal como el uso periódico y frecuente de aerosoles desinfectantes. ${ }^{21}$

Todos los entrevistados consideraron estas infecciones relevantes $\mathrm{y}$, en muchos casos, hubo niños internados a causa de ellas; sin embargo, en una sola institución, había una normativa sobre

TABLA 2. Hábitos de higiene en las salas observadas $(n=12)$

\begin{tabular}{|c|c|c|c|}
\hline Higiene de manos & SÍ & $\%$ & Comentarios \\
\hline Material gráfico sobre el procedimiento & 0 & 0 & * La técnica fue correcta solo en una situación. \\
\hline Situaciones de higiene en adultos, $n=31$ & SÍ & $\%$ & ** En una situación, se utilizó alcohol en gel. \\
\hline Lavados húmedos* & 12 & 39 & $* * *$ En el resto de las situaciones, \\
\hline Secado con papel de uso único & 10 & 31 & se usó toalla de tela o se reutilizó el papel. \\
\hline Uso de alcohol en gel & 19 & 61 & \\
\hline Situaciones de higiene en niños, $n=98$ & SÍ & $\%$ & \\
\hline Lavado húmedo** & 62 & 63 & \\
\hline Secado con papel de uso único*** & 47 & 48 & \\
\hline Higiene con toallas humedecidas & 35 & 36 & \\
\hline Limpieza de secreciones, ${ }^{*} \mathrm{n}=75$ & SÍ & $\%$ & Comentarios \\
\hline Con pañuelo descartable individual & 71 & 95 & * Corresponde a lo observado en \\
\hline Con toallita humedecida & 2 & 3 & situaciones que involucran niños. \\
\hline Con la mano & 2 & 3 & Respecto de los adultos, \\
\hline Descarte correcto del pañuelo & 67 & 89 & las oportunidades observadas fueron 3: \\
\hline Higiene de manos del operador & 3 & 4 & 1 con papel y 2 con el antebrazo. \\
\hline Higiene de manos del niño & 1 & 1 & \\
\hline Cambio de pañal, $\mathrm{n}=85$ & SÍ & $\%$ & Comentarios \\
\hline Uso de guantes (en ambas manos) & 10 & 12 & \\
\hline Uso de 1 guante (en una mano) & 34 & 40 & \\
\hline Sin guantes & 41 & 48 & \\
\hline Descarte inmediato en bolsita individual & 3 & 4 & \\
\hline Descarte inmediato en cesto & 60 & 70 & \\
\hline Lavado de manos del operador & 18 & 21 & \\
\hline Lavado de manos del niño & 0 & 0 & \\
\hline Limpieza posterior del cambiador & 41 & 48 & \\
\hline
\end{tabular}

TABLA 3. Actividades de capacitación y normalizaciones en los jardines participantes $(n=12)$

Capacitación específica para docentes en el último año

1

Realiza actividades de formación para no docentes o padres en el último año 0

Recibió comunicación sobre el tema de entidades oficiales de salud o educación* 4

Tiene guías o normas propias escritas sobre prevención de infecciones respiratorias

Tiene normas o instructivo escrito sobre higiene de manos

Tiene normas escritas para la limpieza del lugar

Tiene registro escrito del cumplimiento de la limpieza

* En los cuatro casos, se trató de un mensaje de correo electrónico cuyo texto consistió en un alerta sobre la llegada del invierno sin recomendaciones específicas de prevención de infecciones. 
medidas de prevención. Si bien, en nuestro país, disponemos de un documento sobre prevención de infecciones en escuelas y jardines maternales dirigido a profesionales de la salud, la mayoría de los jardines visitados no cuenta con uno de ellos como asesor. ${ }^{22}$ Más aún, en aquellos que sí contaban con él, el tema no impresionaba haberse considerado prioritario. La ausencia de guías sobre prevención inespecífica dirigidas al personal de los jardines o de recomendaciones por parte de autoridades educativas o de salud, así como la falta de capacitación al personal, sugieren la necesidad de sostén y supervisión efectivos para poder implementar medidas de prevención en este ámbito. Incluso las situaciones previstas por la Ley 621, como el requerimiento de certificado médico al regreso al jardín luego de una enfermedad o el uso de sectores separados para alimentación y cambio de pañales, no se cumplen en forma regular. Contrariamente a lo que sucede para la prevención de infecciones respiratorias, en la CABA, existe una reglamentación estricta y de amplio cumplimiento referente a cuestiones de seguridad en instituciones educativas, basada en la relevancia de las lesiones no intencionales en la infancia. ${ }^{23}$ La magnitud del problema de las infecciones respiratorias requeriría también de su inclusión prioritaria en la agenda de discusión educativo-sanitaria.

Merece especial atención la dificultad para evitar que los padres dejen en el jardín a niños que están enfermos, que coincide con la encuesta realizada por Sacks ${ }^{24}$ a 1988 directores de jardines maternales de Estados Unidos, quienes manifestaron que la principal medida por mejorar para prevenir las enfermedades infecciosas en su institución era lograr la colaboración de los padres en el cumplimiento de las normativas. Este problema excede el ámbito institucional, ya que una de las funciones sociales de este nivel educativo es permitir a las familias articular el cuidado de los niños con sus obligaciones laborales. ${ }^{25}$ Surge una contradicción entre la capacidad operativa de los centros de cuidado infantil y de su personal, en ausencia de un marco legal que lo rija para brindar cuidados en determinadas condiciones de enfermedad de los niños y cumplir con el rol social antes mencionado. Esta situación afecta especialmente a las familias de nivel socioeconómico más bajo. ${ }^{9,25}$

La lactancia materna es un factor protector frente a infecciones, ${ }^{26,27}$ su promoción es poco costosa y la recepción y manejo de la leche humana por parte del personal del jardín es operativamente simple. Sin embargo, en menos de la mitad de los centros, las madres dejaban leche materna o iban a amamantar a sus hijos.

La educación para la salud en el ámbito educativo es un derecho fundamental de todos los niños. ${ }^{28} \mathrm{La}$ información recogida en este estudio no indica que los centros de cuidado infantil participantes se posicionen en el rol de promotores de salud, en coincidencia con lo descrito por Davó y col. ${ }^{29}$ sobre instituciones educativas del nivel inicial en España. En el pasado, los programas de salud escolar se consideraban responsabilidad exclusiva del sector salud, y las instituciones educativas, como receptoras pasivas de estas intervenciones. El concepto actual, en línea con la Iniciativa Regional Escuelas Promotoras de la Salud (OPS), contempla que los profesionales de salud y de educación pueden y deben ser socios activos en la promoción de hábitos y destrezas para una vida saludable. ${ }^{30}$

Para que las instituciones educativoasistenciales puedan ejercer este rol, es imprescindible un proceso de empoderamiento, que implica desarrollo de competencias y entrenamiento en habilidades. ${ }^{30}$ Este proceso solo puede llevarse a cabo si el tema toma relevancia entre los decisores del ámbito educativoasistencial y ello se traduce en acciones concretas que incluyan generar un marco regulatorio, educar al personal interviniente y difundir a la comunidad en general.

Como limitación de este trabajo, debemos mencionar el hecho de que fue realizado en 12 jardines maternales de gestión privada, que si bien fueron seleccionados a través de una sistemática, no pueden considerarse representativos de todos los jardines de la CABA. Sin embargo, no puede negarse cierta homogeneidad en los resultados, aun cuando es posible considerar la aceptación a participar como un indicador de la importancia percibida del tema.

Un posible sesgo de nuestro estudio está dado por la dificultad para contactar a los responsables de los jardines $y$, en ocasiones, para obtener su aprobación, ya sea por temor a ser denunciados ante autoridades o por un nivel de ocupación que no permitía tomar tiempo para realizar la entrevista. Una especulación posible es que, en algunas instituciones de cuidado infantil, no se percibe como prioritario el tema de la prevención de infecciones. La ausencia de instancias de capacitación, de normativas y de recomendaciones de las autoridades sanitario- 
educativas podría ser condicionante de esta situación.

La fortaleza de este trabajo reside en ser, hasta nuestro conocimiento, el primer aporte de datos locales, necesarios para conocer la envergadura del problema en nuestra comunidad y para emprender acciones dirigidas a mejorar la difusión e implementación de estrategias de prevención, entre los actores implicados.

Son necesarios estudios adicionales para poder hacer inferencias sobre la situación general de los centros de cuidado infantil, en materia de prevención de infecciones, en nuestro medio, así como generar recomendaciones basadas en evidencia e instancias de capacitación específica para el personal de estas instituciones.

\section{CONCLUSIÓN}

Pese a la disponibilidad de recursos y la evidencia existente, la implementación de medidas de prevención de infecciones respiratorias no se realiza en forma regular y sistemática en los jardines visitados.

\section{Agradecimientos}

A las Dras. Mariana Romero, Vera May y Natalia Bikel y a las Lic. Adriana Torres y Diana Vendroc. A las autoridades y personal de los jardines participantes, quienes nos recibieron con calidez y entusiasmo, por su colaboración en este proyecto.

\section{REFERENCIAS}

1. Iwane MK, Edwards KM, Szilagyi PG, Walker FJ, et al. Population-based surveillance for hospitalizations associated with respiratory syncytial virus, influenza virus, and parainfluenza viruses among young children. Pediatrics 2004;113(6):1758-64.

2. Nair H, Simões EA, Rudan I, Gessner BD, et al. Global and regional burden of hospital admissions for severe acute lower respiratory infections in young children in 2010: a systematic analysis. Lancet 2013;381(9875):1380-90.

3. Chaui J, Forlenza R. Infecciones respiratorias agudas sala de situación 2012 Hasta Semana Epidemiológica 26. Departamento de epidemiología delGobierno dela Ciudad de Buenos Aires. [Acceso: 16 de enero de 2015]. Disponible en http://www.neumo-argentina.org/images/articulos_ interes/sala_ira_sem_26-2012.ppt.

4. Ministerio de Salud de la Nación Argentina. Secretaría de Políticas, Regulación e Institutos. Dirección de Estadísticase Información deSalud. Estadísticas Vitales Año: Información básica - Año 2013. [Acceso: 23 de enero de 2015]. Disponible en: http:/ / www.deis.gov.ar/Publicaciones / Archivos / Serie5Nro57.pdf.

5. McCutcheon H, Fitzgerald M. The public health problem of acute respiratory illness in childcare. J Clin Nurs 2001;10(3):305-10.

6. Kamper-Jørgensen M,WohlfahrtJ,SimonsenJ,GrønbaekM, Benn CS. Population-based study of the impact of childcare attendance on hospitalizations for acute respiratory infections. Pediatrics 2006;118(4):1439-46.

7. Repetto F, Diaz Langou G, Aulicino C. Cuidado infantil en la Ciudad Autónoma de Buenos Aires: ¿la disyuntiva entre pañales y pedagogía? Agosto 2012. [Acceso: 10 de octubre de 2014]. Disponible en: http:/ / www.cippec.org/ documents /10179/51827/93+DT+Cuidado+infantil+en+1 $\mathrm{a}+$ Ciudad +Aut $\%$ C3 $\%$ B3noma+de+Buenos+Aires $\% 2 \mathrm{C} \% 20$ Repetto\%2C\%20Diaz+Langou+y+Aulicino\%2C\%202012. pdf/7ab9dc20-490f-4887-a303-5af66ede767d.

8. Rodríguez Enríquez C. La organización del cuidado de niños y niñas en Argentina y Uruguay. Unidad mujer y desarrollo. Santiago de Chile: CEPAL, 2007. (Serie Mujer y Desarrollo $N^{o}$ 90). [Acceso: 10 de octubre de 2014]. Disponible en: http://www.cepal.org/publicaciones / xml/5/32615/lcl2844e.pdf.

9. Zibecchi C. Organizaciones comunitarias y cuidado en la primera infancia: un análisis en torno a las trayectorias, prácticas y saberes de las cuidadoras. Trab Soc 2013(20): 427-47.

10. Serra ME.Prevención deinfecciones respiratorias enjardines maternales: recomendaciones y revisión sistemática de la evidencia. Arch Argent Pediatr 2014;112(4):323-31.

11. Argentina. Ley $n^{\circ}$ 621-Decreto 1089/GCBA/2002. Ley de regulación de habilitación, funcionamiento y supervisión de instituciones privadas de carácter educativo asistencial, destinadas a niños de 45 días a 4 años, no incorporadas a la enseñanza oficial. [Acceso: 14 de enero de 2015]. Disponible en:http:/ / www.buenosaires.gob.ar/dgegp/riea/LEY621. pdf.

12. Ministerio de Educación del Gobierno de la Ciudad de Buenos Aires. Registro de Instituciones Educativo Asistenciales de Gestión Privada. Listado de instituciones con registro definitivo y provisorio. [Acceso: marzo de 2013]. Disponible en: http://www.buenosaires.gob.ar/areas/ educacion/gestion_privada/riae.php?menu_id=23314.

13. Jefferson T, Foxlee R, Del Mar C, Dooley L, et al. Physical interventions to interrupt or reduce the spread of respiratory viruses: systematic review. BMJ 2008;336(7635):77-80.

14. Rabie T, Curtis V. Handwashing and risk of respiratory infections: a quantitative systematic review. Trop Med Int Health 2006;11(3):258-67.

15. Aiello AE, Coulborn RM, Perez V, Larson EL. Effect of Hand Hygiene on Infectious Disease Risk in the Community Setting: A Meta-Analysis. Am J Public Health 2008;98(8):137281.

16. Zomer TP, Erasmus V, Van Beeck EF, Tjon-A-Tsien A, et al. Hand hygiene compliance and environmentaldeterminants in child day care centers: an observational study. Am J Infect Control 2013;41(6):497-502.

17. Barros AJ, Ross DA, Fonseca WV, Williams LA, MoreiraFilho DC. Preventing acute respiratory infections and diarrhoea in child care centers. Acta Paediatr 1999;88(10):1113-8.

18. Wenger JD, Harrison LH, Hightower A, Broome CV. Day care characteristics associated with Haemophilus influenzae disease. Haemophilus influenzae Study Group. Am J Public Health 1990;80(12):1455-8.

19. Cozad A, Jones RD. Disinfection and the prevention of infectious disease. Am J Infect Control 2003;31(4):243-54.

20. Aiello AE, Larson EL. What is the evidence for a causal link between hygiene and infections? Lancet Infect Dis 2002;2(2):103-10.

21. Wolkoff P, Schneider T, Kildesø J, Degerth R, et al. Risk in cleaning: chemical and physical exposure. Sci Total Environ 1998;215(1-2):135-56.

22. Sociedad Argentina de Pediatría. Consenso sobre Prevención de Infecciones en Jardines Maternales y Escuelas. Ciudad Autónoma de Buenos Aires, 1997. [Acceso: 
19 de enero de 2015]. Disponible en: http://www.sap. org.ar/consenso_sobre_prevencion_de_infecciones_en_ jardines_maternales_y_escuelas.php.

23. Ministerio de Educación del Gobierno de la Ciudad de Buenos Aires. Plataforma de escuelas seguras. [Acceso: 19 de enero 2015]. Disponible en: http:/ /www.buenosaires. gob.ar/areas/educacion/gestion_privada/plataformaescuelas_seguras.php?menu_id=34211.

24. Sacks JJ, Addiss DG. The perceived needs of child care center directors in preventing injuries and infectious diseases. Am J Public Health 1995;85(2):266-7.

25. Gheradi N, Pautassi L, Zibecchi C. De eso no se habla: el cuidado en la agenda pública. Estudio de opinión sobre la organización del cuidado. Buenos Aires: Equipo Latinoamericano de Justica y Género. ELA, 2012.

26. Cushing AH, Samet JM, Lambert WE, Skipper BJ, et al. Breastfeeding reduces the risk of respiratory illness in infants. Am J Epidemiol 1998;147(9):863-70.
27. Chantry CJ, Howard CR, Auinger P. Full breastfeeding duration and associated decrease in respiratory tract infection in US children. Pediatrics 2006;117(2):425-32.

28. Organización Panamericana de la Salud. Promoción y educación de la Salud Escolar. Una perspectiva integral: marco conceptual y operativo, taller de capacitación con metodología de diagnóstico y análisis rápido, Red Latinoamericana de Escuelas Promotoras de Salud. Washington DC, 1995.

29. Davó MC, Gil-González D, Vives-Cases C, Álvarez-Dardet C, La Parra D. Las investigaciones sobre promoción y educación para la salud en las etapas de infantil y primaria de la escuela española. Una revisión de los estudios publicados entre 1995 y 2005. Gac Sanit 2008;22(1):58-64.

30. OPS. Ippolito-Shepherd J. Escuelas Promotoras dela Salud. Fortalecimiento de la Iniciativa Regional. Estrategias y líneas de acción 2003-2012. (Serie Promoción de la Salud $\mathrm{N}^{\mathrm{o}}$ 4). Washington DC, 2003. 


\section{Anexo 1.}

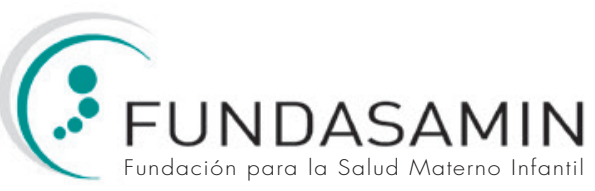

ID de jardín:

Entrevistador:

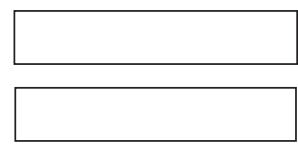

PROYECTO

Prevención de la diseminación de infecciones respiratorias en jardines maternales de la Ciudad Autónoma de Buenos Aires. Diagnóstico de situación.

\section{ENTREVISTA}

\section{A. ASPECTOS GENERALES}

Personal entrevistado:

1. ¿Cuál es su rol en la institución? (marque con cruz lo que corresponda)
Directora
Coordinadora
Maestra de sala
auxiliar
Otra

Con respecto al jardín:

2. Jardín de la Comuna ${ }^{\circ}$ :

3. Gestión: Público $\square$ Privado $\square$

4. ¿Cuánto hace que están en funcionamiento las salas de menores de dos años en este jardín? Años: Meses:

5. ¿Cuántos niños menores de dos años concurren actualmente al jardín?

6. Número de salas para menores de dos años:

Con respecto a los niños que concurren al jardín (a cualquier sala/ de cualquier edad):

7. ¿Ud. cree que las infecciones respiratorias son comunes en esta edad?

$$
\text { Sí } \square \quad \text { No }
$$

8. Ud. considera que las infecciones respiratorias:
a. Son peligrosas para cualquier niño.
b. Son peligrosas para algunos niños particularmente.
c. No son peligrosas para ningún niño.

9. ¿Concurre algún niño con problema crónico de salud o característica especial?:
Sí $\square$
No $\square$
No sabe

10. ¿Algún niño del jardín o hijo del personal del jardín (aunque no concurra a esta institución) ha sido internado por una infección respiratoria este año o el año pasado?
Sí $\square$
¿Cuántos?
No
No sabe

\section{B. CAPACITACIÓN / NORMAS/ PAUTAS DE PREVENCIÓN}

11. ¿Ha recibido durante este año alguna comunicación/indicación/sugerencia por escrito por parte de alguna de las entidades de educación o salud (por ej.: MSAL, GCBA, DGEI).

$$
\text { Sí } \square \text { No }
$$

\section{Comentario:}


12. ¿Hay en su institución algún documento escrito acerca de cómo prevenir las infecciones respiratorias? (propio o adoptado de otra institución).
Sí
No
No sabe

Comentario:

13. ¿Hubo durante el último año en la institución alguna actividad (taller, jornada) para trabajar sobre el tema de infecciones respiratorias?
13.1 Con maestras
Sí $\square$
No $\square$
No sabe
13.2 Con personal no docente
No
No sabe
13.3 Con padres
Sí $\square$
No $\square$
No sabe

14. ¿Implementó la institución algún cambio después de la epidemia de gripe A?
Sí $\square$
14.1 ¿Cuál?

15. Cuando un niño presenta síntomas de infección respiratoria (mocos o tos por ejemplo): ¿Cuál es la conducta de la mayor parte de los padres respecto de la concurrencia al jardín?

a. La mayor parte de los padres lo traen igual.

b. La mayor parte de los padres no lo traen al jardín.

Comentario:

16. Cuando un niño presenta síntomas de infección respiratoria (mocos o tos por ejemplo): ¿Cuál es la conducta institucional respecto de la concurrencia al jardín?

Lo recibimos (es lo que hacemos habitualmente).

No lo recibimos porque hay norma escrita/establecida.

No lo recibimos aunque no hay norma escrita.

Comentario:

17. Si un niño tuvo alguna infección respiratoria como neumonía, bronquiolitis o broncoespasmo. ¿Solicita certificado médico para el regreso al jardín?

Sí $\square$ Siempre $\square \quad$ o a veces: ¿En qué casos?:

18. ¿Ante qué situaciones Uds. llaman a los padres para que retiren a su hijo del jardín?

19. Se lleva actualmente en la institución un registro formal de las ausencias de los niños por motivos de salud:
Sí
No $\square \quad$ No sabe 
20. ¿Cuándo hay indicación formal institucional de que una maestra no ingrese a su puesto de trabajo?

\section{LAVADO DE MANOS}

21. ¿Hay alguna normativa escrita en la institución sobre cuándo deben lavarse las manos las maestras? Sí $\square$ No

22. ¿Hay alguna normativa escrita en su institución sobre cuándo debe lavarse las manos el personal no docente?

Sí $\square \quad$ No

23. ¿Hay alguna normativa escrita en la institución sobre cuándo deben lavarse las manos los niños menores de dos años?

Sí $\square \quad$ No

D. INMUNIZACIONES /CONTROLES DE SALUD

24. ¿La institución sigue alguna normativa con respecto al control de la vacunación de los niños?

Sí $\square$ No

Comentario:

25. ¿La institución sigue alguna normativa con respecto al control de la vacunación del personal del jardín?

Sí $\square \quad$ No

Comentario:

E. LACTANCIA

26. ¿Alguna madre deja leche materna para alimentar al niño?
Sí $\square$ ¿Cuántas estima?
No $\square$
No sabe

27. ¿Viene alguna madre a amamantar al jardín?
Sí
No
No sabe

\section{F. INFRAESTRUCTURA/LIMPIEZA}

28. ¿Hay algún sector para fumadores en la institución?

Sí $\square \quad$ No $\square \quad$ No sabe

29. ¿La institución cuenta con un pediatra, enfermera u otro profesional que se desempeñe como asesor en temas de salud?

Sí $\square \quad$ No $\square \quad$ No sabe

En la institución

Al llamado

Eventual (consultas) 
IV / Arch Argent Pediatr 2015;113(3):205-212 / Artículo original

30. ¿De qué forma tienen organizada la limpieza del lugar?
a. Instructivo escrito Sí
No $\square$
No sabe
b. Registro escrito
Sí $\square$
No $\square$
No sabe

31. Ropa de cuna individual

Sí $\square \quad$ No

32. ¿Dónde se realiza el lavado de mantas, acolchados, fundas de huevito?

33. ¿Cada cuánto se realiza ese lavado?

34. ¿Cómo se realiza la higiene de los juguetes?

35. ¿Quiere hacer algún comentario? 


\section{Anexo 2.}

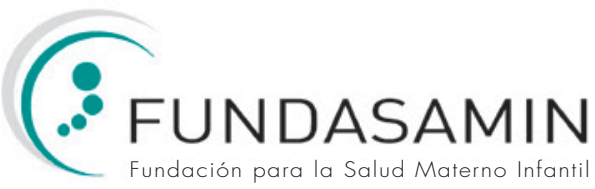

ID de jardín:

Entrevistador:

Prevención de la diseminación de infecciones respiratorias en jardines maternales de la Ciudad Autónoma de Buenos Aires. Diagnóstico de situación

\section{GUÍA DE OBSERVACIÓN}

\section{A. INFRAESTRUCTURA Y RECURSOS DE LA SALA}

Sala $n^{\circ}$ :

1. Cantidad de niños que están presentes en la sala:

2. Edad de los niños: $\quad$ Lactantes $\square \quad$ Deambuladores $\square$

3. Cantidad de maestrasy auxiliares docentes que están presentes en la sala:

4. Número de ventanas:

5. Número de ventanas fijas:

6. Aire acondicionado:
Sí
No $\square$

7. Tipo de calefacción:

Eléctrica $\square \quad$ A gas $\square \quad$ Combustión $\square \quad$ Por aire acondicionado

8. Piso:

Lavable (cerámico, cemento, linóleo, plastificado, etc.)

Parquet o pinotea (encerado)

Baldosón tipo patio (limpieza con kerosén)

Alfombra

Otro:

9. Presencia de alfombras removibles:

Sí $\square \quad$ No

10. Pileta para uso de esta sala:

En la sala $\square \quad$ Contiguo a la sala $\square \quad$ En otro lugar del jardín

11. Tipo de canilla: Monocomando $\square \quad$ Accionada a mano

12. Elementos para lavado de manos:

Jabón líquido

Jabón en barra

Antiséptico jabonoso

Otro

Especificar: 
13. Elementos de secado:

Toallas de papel de uso individual

Rollo de cocina

Toallas de tela

Secador eléctrico

14. Dispositivo con alcohol gel:

Sí $\square \quad$ No $\square \quad$ ¿Cuántos? $\square \quad$ ¿Dónde?

15. Cremas de manos:

Sí $\square \quad$ ¿En dispositivo expendedor? Sí $\square \quad$ No

No

16. Descartador de residuos:
Con tapa
Sí
Accionado a pedal Sí $\square$
No
No

17. Tipo de cuna:

Acrílico

Cromada

Madera

Metal

Moisés

Otro

18. Cantidad de cunas:

19. ¿Hay almohadas?

$\begin{array}{lll}\text { Sí } \square \quad \text { No } & \square \\ \text { Individual } & \text { Sí } \square & \text { No } \square \\ \text { Lavable } & \text { Sí } \square & \text { No } \square\end{array}$

20. ¿Hay almohadones?

Sí $\square \quad$ No $\square$

Individual Sí $\square$

Lavable Sí

No

No

21. ¿Hay Colchonetas?
Sí
No
Individual
Sí $\square$
Lavable
Sí
No $\square$
No $\square$

22. Hay sectores bien diferenciados para

Pañales $\square \quad$ Alimentación

23. Elementos de higiene (oleo calcáreo, toallitas, etc.)

Individual: $\quad$ Sí $\square \quad$ No $\square$

24. ¿Hay pañuelosdescartables a la vista o fácilmente disponibles?

Sí $\square \quad$ No $\square$

25. ¿Hay guantes?

Sí $\square \quad$ No $\square$

26. ¿Hay bolsitas?

Sí $\square \quad$ No $\square$ 


\section{B. OBSERVACIÓN DE HÁBITOS DE HIGIENE:}

Todo lo que no pueda ser observado (independientemente del motivo) rotule con la leyenda "no se observa".

B.a. Uso de uniforme

27. ¿Cómo es la indumentaria usual para trabajar en la sala? Describir ropa y calzado.

\section{B.b. Lavado de manos}

28. ¿Hay en la institución alguna indicación/capacitación o material educativo visible sobre cómo lavarse las manos?

Sí $\square \quad$ No $\square$

29. ¿Hay alguna maestra o personal en contacto directo con los niños que esté usando accesorios de moda que dificulten la higiene de manos (tales como pulseras, relojes, anillos con relieve o uñas postizas)?

$$
\text { Sí } \square \quad \text { No }
$$

\section{Lavado de manos}

\begin{tabular}{|c|c|c|c|c|c|c|c|c|}
\hline Observación & Húmedo & Otro & Enjabonado & Frotado & Enjuague & Secado & Oportunidad & Comentario \\
\hline \multicolumn{9}{|l|}{1} \\
\hline \multicolumn{9}{|l|}{2} \\
\hline \multicolumn{9}{|l|}{3} \\
\hline \multicolumn{9}{|l|}{4} \\
\hline \multicolumn{9}{|l|}{5} \\
\hline \multicolumn{9}{|l|}{6} \\
\hline \multicolumn{9}{|l|}{7} \\
\hline \multicolumn{9}{|l|}{8} \\
\hline \multicolumn{9}{|l|}{9} \\
\hline \multicolumn{9}{|l|}{10} \\
\hline \multicolumn{9}{|l|}{11} \\
\hline \multicolumn{9}{|l|}{12} \\
\hline \multicolumn{9}{|l|}{13} \\
\hline \multicolumn{9}{|l|}{$\overline{14}$} \\
\hline \multicolumn{9}{|l|}{15} \\
\hline \multicolumn{9}{|l|}{16} \\
\hline \multicolumn{9}{|l|}{17} \\
\hline \multicolumn{9}{|l|}{18} \\
\hline \multicolumn{9}{|l|}{19} \\
\hline \multicolumn{9}{|l|}{20} \\
\hline \multicolumn{9}{|l|}{21} \\
\hline \multicolumn{9}{|l|}{22} \\
\hline \multicolumn{9}{|l|}{23} \\
\hline \multicolumn{9}{|l|}{24} \\
\hline \multicolumn{9}{|l|}{25} \\
\hline \multicolumn{9}{|l|}{26} \\
\hline \multicolumn{9}{|l|}{27} \\
\hline 28 & & & & & & & & \\
\hline 29 & & & & & & & & \\
\hline 30 & & & & & & & & \\
\hline 31 & & & & & & & & \\
\hline 32 & & & & & & & & \\
\hline 33 & & & & & & & & \\
\hline 34 & & & & & & & & \\
\hline 35 & & & & & & & & \\
\hline
\end{tabular}


VIII / Arch Argent Pediatr 2015;113(3):205-212 / Artículo original

\section{B.c. Limpieza de secreciones}

\begin{tabular}{|c|c|c|c|c|c|c|c|}
\hline \multicolumn{8}{|c|}{ Manejo de secreciones } \\
\hline Observación & Catarro & Tos & Secreciones & Higiene & Tiempo & Descarte en & Comentario \\
\hline \multicolumn{8}{|l|}{1} \\
\hline \multicolumn{8}{|l|}{2} \\
\hline \multicolumn{8}{|l|}{3} \\
\hline \multicolumn{8}{|l|}{4} \\
\hline \multicolumn{8}{|l|}{5} \\
\hline \multicolumn{8}{|l|}{6} \\
\hline \multicolumn{8}{|l|}{7} \\
\hline \multicolumn{8}{|l|}{8} \\
\hline \multicolumn{8}{|l|}{9} \\
\hline \multicolumn{8}{|l|}{10} \\
\hline \multicolumn{8}{|l|}{11} \\
\hline \multicolumn{8}{|l|}{12} \\
\hline \multicolumn{8}{|l|}{13} \\
\hline \multicolumn{8}{|l|}{14} \\
\hline \multicolumn{8}{|l|}{15} \\
\hline \multicolumn{8}{|l|}{16} \\
\hline \multicolumn{8}{|l|}{17} \\
\hline \multicolumn{8}{|l|}{18} \\
\hline \multicolumn{8}{|l|}{19} \\
\hline \multicolumn{8}{|l|}{20} \\
\hline \multicolumn{8}{|l|}{21} \\
\hline \multicolumn{8}{|l|}{22} \\
\hline \multicolumn{8}{|l|}{23} \\
\hline \multicolumn{8}{|l|}{24} \\
\hline \multicolumn{8}{|l|}{25} \\
\hline \multicolumn{8}{|l|}{26} \\
\hline 27 & & & & & & & \\
\hline 28 & & & & & & & \\
\hline 29 & & & & & & & \\
\hline 30 & & & & & & & \\
\hline 31 & & & & & & & \\
\hline 32 & & & & & & & \\
\hline 33 & & & & & & & \\
\hline 34 & & & & & & & \\
\hline 35 & & & & & & & \\
\hline
\end{tabular}

\section{LACTANCIA}

69. ¿Hay visible difusión o propaganda de fomento y / o apoyo de la lactancia materna? Sí $\square \quad$ No $\square$

70. ¿Hay un espacio especialmente destinado para que las madres amamanten? Sí $\square$ No $\square$

71. ¿Hay alguna madre amamantando?

$$
\text { Sí } \square \quad \text { No } \square
$$


Prevención de infecciones respiratorias. Situación en 12 jardines maternales privados de la Ciudad Autónoma de Buenos Aires / IX

\section{CAPACITACIÓN ESPECÍFICA DE LAS MAESTRAS DE LA SALA}

Recibió capacitación específica durante el último año

Docente 1

Docente 2

Docente 3

Docente 4

\section{E. CAMBIOS DE PAÑALES}

\begin{tabular}{|c|c|c|c|c|c|c|c|c|}
\hline \multicolumn{9}{|c|}{ Cambios de pañales } \\
\hline Observación & $\begin{array}{c}\text { Con } \\
\text { guantes }\end{array}$ & $\begin{array}{c}\text { Un } \\
\text { guante }\end{array}$ & $\begin{array}{c}\text { Sin } \\
\text { guante }\end{array}$ & $\begin{array}{c}\text { Descarte } \\
\text { en }\end{array}$ & $\begin{array}{c}\text { Lavado de } \\
\text { manos operador }\end{array}$ & $\begin{array}{c}\text { Lavado de } \\
\text { manos niño }\end{array}$ & $\begin{array}{c}\text { Limpieza del } \\
\text { cambiador }\end{array}$ & Comentario \\
\hline \multicolumn{9}{|l|}{1} \\
\hline \multicolumn{9}{|l|}{2} \\
\hline \multicolumn{9}{|l|}{3} \\
\hline \multicolumn{9}{|l|}{4} \\
\hline \multicolumn{9}{|l|}{5} \\
\hline \multicolumn{9}{|l|}{6} \\
\hline \multicolumn{9}{|l|}{7} \\
\hline \multicolumn{9}{|l|}{8} \\
\hline \multicolumn{9}{|l|}{9} \\
\hline \multicolumn{9}{|l|}{10} \\
\hline \multicolumn{9}{|l|}{11} \\
\hline \multicolumn{9}{|l|}{12} \\
\hline \multicolumn{9}{|l|}{13} \\
\hline \multicolumn{9}{|l|}{14} \\
\hline \multicolumn{9}{|l|}{15} \\
\hline \multicolumn{9}{|l|}{16} \\
\hline \multicolumn{9}{|l|}{17} \\
\hline \multicolumn{9}{|l|}{18} \\
\hline \multicolumn{9}{|l|}{19} \\
\hline \multicolumn{9}{|l|}{20} \\
\hline \multicolumn{9}{|l|}{21} \\
\hline \multicolumn{9}{|l|}{22} \\
\hline \multicolumn{9}{|l|}{23} \\
\hline \multicolumn{9}{|l|}{24} \\
\hline \multicolumn{9}{|l|}{25} \\
\hline \multicolumn{9}{|l|}{26} \\
\hline \multicolumn{9}{|l|}{27} \\
\hline 28 & & & & & & & & \\
\hline 29 & & & & & & & & \\
\hline 30 & & & & & & & & \\
\hline 31 & & & & & & & & \\
\hline
\end{tabular}

